University of Nebraska - Lincoln

DigitalCommons@University of Nebraska - Lincoln

\title{
$10-15-1975$
}

\section{Path-integral formulation of scattering theory}

\author{
Paul Finkler \\ University of Nebraska-Lincoln, pfinkler1@unl.edu \\ C. Edward Jones \\ University of Nebraska-Lincoln \\ M. Misheloff \\ University of Nebraska-Lincoln
}

Follow this and additional works at: https://digitalcommons.unl.edu/physicsfinkler

Part of the Physics Commons

Finkler, Paul; Jones, C. Edward; and Misheloff, M., "Path-integral formulation of scattering theory" (1975). Paul Finkler Papers. 11.

https://digitalcommons.unl.edu/physicsfinkler/11

This Article is brought to you for free and open access by the Research Papers in Physics and Astronomy at DigitalCommons@University of Nebraska - Lincoln. It has been accepted for inclusion in Paul Finkler Papers by an authorized administrator of DigitalCommons@University of Nebraska - Lincoln. 


\title{
Path-integral formulation of scattering theory
}

\author{
W. B. Campbell*' \\ Kellogg Radiation Laboratory, California Institute of Technology, Pasadena, California 91125 \\ P. Finkler, C. E. Jones, ${ }^{\ddagger}$ and M. N. Misheloff ${ }^{\ddagger}$ \\ Behlen Laboratory of Physics, University of Nebraska, Lincoln, Nebraska 68508
}

(Received 14 July 1975)

\begin{abstract}
A new formulation of nonrelativistic scattering theory is developed which expresses the $S$ matrix as a path integral. This formulation appears to have at least two advantages: (1) A closed formula is obtained for the $S$ matrix in terms of the potential, not involving a series expansion; (2) the energy-conserving $\delta$ function can be explicitly extracted using a technique analogous to that of Faddeev and Popov, thereby yielding a closed pathintegral expression for the $T$ matrix. The introduction of the concept of the classical interaction picture provides considerable physical insight into this formulation. This formulation also suggests a successionof improvements to the eikonal approximation, the first of which is discussed explicitly.
\end{abstract}

\section{INTRODUCTION}

Feynman's path-integral formulation of quantum mechanics ${ }^{1}$ has been a subject of considerable interest recently. ${ }^{2}$ In this paper, we develop a pathintegral formulation of nonrelativistic scattering theory. This work is an application of previous results of a path-integral formulation for arbitrary generators. $^{3}$

Starting from the quantum-mechanical interaction picture, in Sec. II we derive an explicit pathintegral representation of the $S$ matrix. In Sec. III we discuss classical scattering processes in a set of canonical variables which we call the classical interaction picture. Particular attention is paid to the form that time translation invariance takes in the classical interaction picture. The relationship between the path-integral representation of the $S$ matrix and the classical interaction picture "action" is obtained. In Sec. IV, we derive the Born series for the $T$ matrix using our formalism. In Sec. V, we use a technique similar to that of Faddeev and Popov ${ }^{4}$ to extract the energy conservation $\delta$ function from the $S$ matrix. We thereby obtain an explicit path-integral expression for the $T$ matrix. The form of this expression is suggestive of the eikonal approximation. In Sec.
VI, we discuss the eikonal approximation from the point of view of our formalism and propose a simple improvement.

\section{PATH-INTEGRAL REPRESENTATION OF THE $S$ MATRIX}

We consider the scattering of a particle of mass $m$ by a potential $V(q)$ which approaches zero faster than $|q|^{-1}$ at large distances. The $S$ matrix is given by

$$
S\left(\overrightarrow{\mathrm{p}}^{\prime}, \overrightarrow{\mathrm{p}}\right)=\lim _{\tau \rightarrow \infty}\left\langle\overrightarrow{\mathrm{p}}^{\prime}\left|U_{I}(\tau,-\tau)\right| \overrightarrow{\mathrm{p}}\right\rangle,
$$

where $U_{I}$ is the interaction-picture time-development operator. It satisfies the integral equation

$$
U_{I}\left(t^{\prime}, t\right)=I-i \int_{t}^{t^{\prime}} d t^{\prime \prime} H_{I}\left(t^{\prime \prime}\right) U_{I}\left(t^{\prime \prime}, t\right),
$$

where

$$
H_{I}(t)=\exp \left(i \frac{p^{2}}{2 m} t\right) V(\overrightarrow{\mathrm{q}}) \exp \left(-i \frac{p^{2} t}{2 m}\right) .
$$

In order to derive a path-integral expression for $S$, we imagine the time interval between $-\tau$ and $\tau$ as being broken up into a large number of smaller time intervals. The matrix element of $U_{I}$ can be written as

$$
\left\langle\overrightarrow{\mathrm{p}}^{\prime}\left|U_{I}(\tau,-\tau)\right| \overrightarrow{\mathrm{p}}\right\rangle=\int d^{3} p_{1} \cdots d^{3} p_{N-1}\left\langle\overrightarrow{\mathrm{p}}_{N}\left|U_{I}\left(t_{N}, t_{N-1}\right)\right| \overrightarrow{\mathrm{p}}_{N-1}\right\rangle\left\langle\overrightarrow{\mathrm{p}}_{N-1}\left|U_{I}\left(t_{N-1}, t_{N-2}\right)\right| \overrightarrow{\mathrm{p}}_{N-2}\right\rangle \cdots\left\langle\overrightarrow{\mathrm{p}}_{1}\left|U_{I}\left(t_{1}, t_{0}\right)\right| \overrightarrow{\mathrm{p}}_{0}\right\rangle
$$

where

$$
t_{i}=-\tau+\frac{2 \tau}{N} i, \quad \overrightarrow{\mathrm{p}}_{N}=\overrightarrow{\mathrm{p}}^{\prime}, \quad \overrightarrow{\mathrm{p}}_{0}=\overrightarrow{\mathrm{p}}
$$

For very large $N$, we may use Eq. (II.2) once-iterated and (II.3) to approximate $\left\langle\overrightarrow{\mathrm{p}}_{i+1}\left|U_{I}\left(t_{i+1}, t_{i}\right)\right| \overrightarrow{\mathrm{p}}_{i}\right\rangle$ by 


$$
\begin{aligned}
\left\langle\overrightarrow{\mathrm{p}}_{i+1}\left|U_{I}\left(t_{i+1}, t_{i}\right)\right| \overrightarrow{\mathrm{p}}_{i}\right\rangle \approx\left\langle\overrightarrow{\mathrm{p}}_{i+1}\right| I- & i \frac{2 \tau}{N} \exp \left(i \frac{p_{i+1}^{2}}{2 m} t_{i}\right) V(\overrightarrow{\mathrm{q}}) \exp \left(-i \frac{p_{i}^{2}}{2 m} t_{i}\right)\left|\overrightarrow{\mathrm{p}}_{i}\right\rangle \\
= & \int \frac{d^{3} q_{i+1}}{(2 \pi)^{3}} \exp \left[-i \overrightarrow{\mathrm{q}}_{i+1} \cdot\left(\overrightarrow{\mathrm{p}}_{i+1}-\overrightarrow{\mathrm{p}}_{i}\right)\right] \\
& -i \int \frac{d^{3} q_{i+1}}{(2 \pi)^{3}} \frac{2 \tau}{N} \exp \left\{-i\left[\overrightarrow{\mathrm{q}}_{i+1} \cdot\left(\overrightarrow{\mathrm{p}}_{i+1}-\overrightarrow{\mathrm{p}}_{i}\right)-\frac{1}{2 m}\left({p_{i+1}}^{2}-p_{i}{ }^{2}\right) t_{i}\right]\right\} V\left(\overrightarrow{\mathrm{q}}_{i+1}\right) .
\end{aligned}
$$

In the second integral, we may change the variable of integration from $\overrightarrow{\mathrm{q}}_{i+1}$ to $\overrightarrow{\mathrm{q}}_{i+1}-(1 / 2 m)\left(\overrightarrow{\mathrm{p}}_{i+1}+\overrightarrow{\mathrm{p}}_{i}\right) t_{i}$. Combining the two resulting integrals and reexponentiating, we obtain

$$
\left\langle\overrightarrow{\mathrm{p}}_{i+1}\left|U_{I}\left(t_{i+1}, t_{i}\right)\right| \overrightarrow{\mathrm{p}}_{i}\right\rangle \approx \int \frac{d^{3} q_{i+1}}{(2 \pi)^{3}} \exp \left\{-i\left[\overrightarrow{\mathrm{q}}_{i+1} \cdot\left(\overrightarrow{\mathrm{p}}_{i+1}-\overrightarrow{\mathrm{p}}_{i}\right)+\frac{2 \tau}{N} V\left(\overrightarrow{\mathrm{q}}_{i+1}+\frac{\overrightarrow{\mathrm{p}}_{i+1}+\overrightarrow{\mathrm{p}}_{i}}{2 m} t_{i}\right)\right]\right\} \text {. }
$$

We now combine Eqs. (II.1), (II.4), and (II.7) to obtain a path-integral representation for the $S$ matrix given by

$$
\begin{aligned}
S\left(\overrightarrow{\mathrm{p}}^{\prime}, \overrightarrow{\mathrm{p}}\right) & =\int\left[d^{3} p(t)\right]\left[\frac{d^{3} q(t)}{2 \pi^{3}}\right] \exp \left\{-i \int_{-\infty}^{\infty} d t\left[\overrightarrow{\mathrm{q}} \cdot \frac{d \overrightarrow{\mathrm{p}}}{d t}+V\left(\overrightarrow{\mathrm{q}}+\frac{\overrightarrow{\mathrm{p}}}{m} t\right)\right]\right\} \\
& =\lim _{\tau \rightarrow \infty} \lim _{N \rightarrow \infty} \int \prod_{i=1}^{N-1} d^{3} p_{i} \prod_{i=1}^{N} \frac{d^{3} q_{i+1}}{(2 \pi)^{3}} \exp \left\{-i \sum_{j=1}^{N}\left[\overrightarrow{\mathrm{q}}_{j} \cdot\left(\overrightarrow{\mathrm{p}}_{j}-\overrightarrow{\mathrm{p}}_{j-1}\right)+\frac{2 \tau}{N} V\left(\overrightarrow{\mathrm{q}}_{j}+\frac{\overrightarrow{\mathrm{p}}_{j}+\overrightarrow{\mathrm{p}}_{j-1}}{2 m} t_{j-1}\right)\right]\right\},
\end{aligned}
$$

where $t_{j}, p_{0}$, and $p_{N}$ are given by Eq. (II.5). As we shall see in Sec. IV, the appearance of the combination $\overrightarrow{\mathrm{p}}_{j}+\overrightarrow{\mathrm{p}}_{j-1}$ in Eq. (II.8) is crucial both for energy conservation and for the appearance of energy denominators in perturbation theory.

Equation (II.8) is the basic result of this section. This form for the $S$ matrix is particularly suitable for the extraction of an energy-conserving $\delta$ function; we shall exploit this fact in Sec. V. We remark that alternative path-integral expressions for the $S$ matrix can easily be derived. For example, if we did not make the change of variables leading to Eq. (II.7), we would have obtained

$$
S\left(\overrightarrow{\mathrm{p}}^{\prime}, \overrightarrow{\mathrm{p}}\right)=\lim _{\tau \rightarrow \infty} \lim _{N \rightarrow \infty} \int \prod_{i=1}^{N-1} d^{3} p_{i} \prod_{i=1}^{N} \frac{d^{3} q_{i}}{(2 \pi)^{3}} \exp \left\{-i \sum_{j=1}^{N}\left[\overrightarrow{\mathrm{q}}_{j} \cdot\left(\overrightarrow{\mathrm{p}}_{j}-\overrightarrow{\mathrm{p}}_{j-1}\right)+\frac{2 \tau}{N} \exp \left(i\left(p_{j}{ }^{2}-p_{j-1}{ }^{2}\right) \frac{t_{j-1}}{2 m}\right) V\left(\overrightarrow{\mathrm{q}}_{i}\right)\right]\right\} .
$$

Alternatively, the change of variables $q_{i} \rightarrow q_{i}+\left[\left(p_{i+1}+p_{i}\right) / 2 m\right] t_{i}$ in Eq. (II.8) leads to the result

$$
\begin{aligned}
S\left(\overrightarrow{\mathrm{p}}^{\prime}, \overrightarrow{\mathrm{p}}\right) & =\lim _{\tau \rightarrow \infty} \exp \left(i \frac{p^{2}+p^{2}}{2 m} \tau\right) \int\left[d^{3} p(t)\right]\left[\frac{d^{3} q(t)}{(2 \pi)^{3}}\right] \exp \left[-i \int_{-\infty}^{\infty} d t\left(\overrightarrow{\mathrm{q}} \cdot \frac{d \overrightarrow{\mathrm{p}}}{d t}+\frac{\overrightarrow{\mathrm{p}}^{2}}{2 m}+V(\overrightarrow{\mathrm{q}})\right)\right] \\
& \equiv \lim _{\tau \rightarrow \infty} \exp \left(i \frac{p^{2}+p^{\prime 2}}{2 m} \tau\right) \lim _{N \rightarrow \infty} \int \prod_{i=1}^{N-1} d^{3} p_{i} \prod_{i=1}^{N} \frac{d^{3} q_{i}}{(2 \pi)^{3}} \exp \left\{-i \sum_{j=1}^{N}\left[\overrightarrow{\mathrm{q}}_{j} \cdot\left(\overrightarrow{\mathrm{p}}_{j}-\overrightarrow{\mathrm{p}}_{j-1}\right)+\frac{2 \tau}{N}\left(\frac{p_{j}^{2}}{2 m}+V\left(\overrightarrow{\mathrm{q}}_{j}\right)\right)\right]\right\} .
\end{aligned}
$$

\section{CLASSICAL INTERACTION PICTURE}

Considerable insight into Eq. (II.8) may be obtained by a consideration of classical scattering processes in a set of canonical variables which we shall call the classical interaction picture. These variables have the important feature that in a scattering process all of them approach constants as $t$ approaches $\pm \infty$, whereas the actual Cartesian position variables diverge in this limit. The values of the interaction picture variables in the asymptotic limit can thus be conveniently used to specify unique scattering trajectories.

We consider a classical Hamiltonian given by

$$
H(\overrightarrow{\mathrm{Q}}, \overrightarrow{\mathrm{P}})=\frac{\overrightarrow{\mathrm{P}}^{2}}{2 m}+V(Q) .
$$

For this Hamiltonian, the momentum and position satisfy the familiar equations of motion:

$$
\frac{d \overrightarrow{\mathrm{P}}}{d t}=-\vec{\nabla} V(\overrightarrow{\mathrm{Q}})
$$

and

$$
\frac{d \overrightarrow{\mathrm{Q}}}{d t}=\frac{\overrightarrow{\mathrm{P}}(t)}{m} .
$$

We define the classical interaction picture variables $\overrightarrow{\mathrm{p}}(t)$ and $\overrightarrow{\mathrm{q}}(t)$ in terms of $\overrightarrow{\mathrm{P}}(t)$ and $\vec{Q}(t)$ by the 
equations

$$
\overrightarrow{\mathrm{p}}(t)=\overrightarrow{\mathrm{P}}(t)
$$

and

$$
\overrightarrow{\mathrm{q}}(t)=\overrightarrow{\mathrm{Q}}(t)-\frac{\overrightarrow{\mathrm{P}}(t)}{m} t .
$$

In terms of $\vec{p}$ and $\vec{q}$, the classical equations of motion are given by

$$
\frac{d \overrightarrow{\mathrm{p}}}{d t}=-\vec{\nabla} V\left(\overrightarrow{\mathrm{q}}+\frac{\overrightarrow{\mathrm{p}}}{m} t\right)
$$

and

$$
\frac{d \overrightarrow{\mathrm{q}}}{d t}=\frac{t}{m} \vec{\nabla} V\left(\overrightarrow{\mathrm{q}}+\frac{\overrightarrow{\mathrm{p}}}{m} t\right) .
$$

These equations imply that $\vec{p}(t)$ and $\vec{q}(t)$ satisfy a modified Hamilton's principle for which the "action" is given by

$$
\tilde{A}=-\int d t\left[\overrightarrow{\mathrm{q}} \cdot \frac{d \overrightarrow{\mathrm{p}}}{d t}+V\left(\overrightarrow{\mathrm{q}}+\frac{\overrightarrow{\mathrm{p}}}{m} t\right)\right],
$$

where the variation of the end-point $\vec{p}$ 's are constrained to zero.

Comparing Eq. (II.8) with Eq. (III.5), we note that the weighting factor for each phase-space path in the path-integral expression for $S\left(\vec{p}^{\prime}, \vec{p}\right)$ is given by $e^{i \hat{A}}$. This situation is analogous to that for the path-integral expression of the positionspace Green's function, $K\left(\overrightarrow{\mathrm{q}}_{f}, l_{f} ; \overrightarrow{\mathrm{q}}_{i}, t_{i}\right)$, for which the weighting factor is given by $e^{i A}$, where the action $A$ is given by

$$
A=\int d t\left[\overrightarrow{\mathrm{p}} \cdot \frac{d \overrightarrow{\mathrm{q}}}{d t}-H(\overrightarrow{\mathrm{q}}, \overrightarrow{\mathrm{p}})\right] \text {. }
$$

A quantum-mechanical scattering process is completely described by the $S$ matrix which is a function of the initial and final momenta. In contrast, the initial and final momenta do not com- pletely determine a classical scattering process; in particular, they do not determine the trajectory of a particle (i.e., the position and momentum as functions of time). The crucial point is that time translational invariance implies that if $\vec{Q}(t)$ and $\overrightarrow{\mathrm{P}}(t)$ are solutions of Eq. (III.2) which satisfy the boundary conditions $\lim _{t \rightarrow-\infty} \overrightarrow{\overrightarrow{\mathbf{P}}}(t)=\overrightarrow{\mathbf{P}}_{i}$ and $\lim _{t \rightarrow+\infty} \overrightarrow{\mathbf{P}}(t)$ $=\overrightarrow{\mathrm{P}}_{f}$, so are $\overrightarrow{\mathrm{Q}}(t+T)$ and $\overrightarrow{\mathrm{P}}(t+T)$ for any fixed $T$. Alternatively, a classical trajectory is specified by six independent boundary conditions. The constraint of energy conservation implies that $\overrightarrow{\mathbf{P}}_{i}$ and $\overrightarrow{\mathrm{P}}_{f}$ can supply only five of these; one additional boundary condition must be specified in order to uniquely determine the trajectory.

The classical interaction picture provides a particularly convenient framework for introducing this additional boundary condition. To see this, we first note that for potentials which fall off faster than $1 / r$, not only does $\overrightarrow{\mathrm{p}}(t)$ approach a constant as $t \rightarrow+\infty$ or $t \rightarrow-\infty$, but so does $\overrightarrow{\mathrm{q}}(t)$. Furthermore, time translational invariance assumes the form that if $\vec{q}(t)$ and $\vec{p}(t)$ are solutions of Eq. (III.4) which satisfy the boundary conditions $\lim _{t \rightarrow-\infty} \overrightarrow{\mathrm{P}}(t)=\overrightarrow{\mathrm{P}}_{i}$ and $\lim _{t \rightarrow+\infty} \overrightarrow{\mathrm{P}}(t)=\overrightarrow{\mathrm{P}}_{f}$, so are $\overrightarrow{\mathrm{q}}(t+T)+[\overrightarrow{\mathrm{p}}(t+T) / m \mid T$ and $\overrightarrow{\mathrm{p}}(t+T)$ for any fixed $T$. Thus, a finite time translation also translates the initial (or final) $\vec{q}$ by a vector which is parallel to the initial (or final) momentum; this implies that different trajectories which are related to each other by finite time translations have different values of $\vec{q}(-\infty) \cdot \vec{p}(-\infty)$ [or $\overrightarrow{\mathrm{q}}(+\infty) \cdot \overrightarrow{\mathrm{p}}(+\infty)$ ]. The additional boundary condition needed to specify the trajectory can therefore be chosen by specifying the value of $\overrightarrow{\mathrm{q}}(-\infty) \cdot \overrightarrow{\mathrm{p}}(-\infty)[$ or $\overrightarrow{\mathrm{q}}(+\infty) \cdot \overrightarrow{\mathrm{p}}(+\infty)$ ].

The analog of the above considerations can be used to explicitly extract the energy conservation $\delta$ function from the $S$ matrix. We shall discuss this further in Sec. V and thereby find a path-integral representation of the scattering amplitude.

\section{BORN EXPANSION}

The Born expansion can easily be obtained from Eq. (II.8). We begin by expanding Eq. (II.8) in a power series in the potential:

$$
S\left(\overrightarrow{\mathrm{p}}^{\prime}, \overrightarrow{\mathrm{p}}\right)=\sum_{n=0}^{\infty} \frac{(-i)^{n}}{n !} \int\left[d^{3} p(t)\right]\left[\frac{d^{3} q(t)}{(2 \pi)^{3}}\right] \exp \left(-i \int_{-\infty}^{\infty} d t \overrightarrow{\mathrm{q}} \cdot \frac{d \overrightarrow{\mathrm{p}}}{d t}\right) \int_{-\infty}^{\infty} d t_{1} V\left(\overrightarrow{\mathrm{q}}\left(t_{1}\right)+\frac{\overrightarrow{\mathrm{p}}\left(t_{1}\right)}{m} t_{1}\right) \cdots \int_{-\infty}^{\infty} d t_{n} V\left(\overrightarrow{\mathrm{q}}\left(t_{n}\right)+\frac{\overrightarrow{\mathrm{p}}\left(t_{n}\right)}{m} t_{n}\right) \cdot
$$

In the $n$th term, we interchange the order of integration over the times $t_{1}, \ldots, t_{n}$ and the path. We furthermore change variables in the time integrals such that they are done in chronological sequence. These manipulations yield

$$
\begin{aligned}
S\left(\overrightarrow{\mathrm{p}}^{\prime}, \overrightarrow{\mathrm{p}}\right)=\sum_{n=0}^{\infty}(-i)^{n} \int_{-\infty}^{\infty} d t_{n} \int_{-\infty}^{t_{n}} d t_{n-1} \cdots \int_{-\infty}^{t_{2}} d t_{1} \int\left[d^{3} p(t)\right]\left[\frac{d^{3} q(t)}{(2 \pi)^{3}}\right] \exp \left(-i \int_{-\infty}^{\infty} d t \overrightarrow{\mathrm{q}} \cdot \frac{d \overrightarrow{\mathrm{p}}}{d t}\right) \\
\times V\left(\overrightarrow{\mathrm{q}}\left(t_{1}\right)+\frac{\overrightarrow{\mathrm{p}}\left(t_{1}\right)}{m} t_{1}\right) \cdots V\left(\overrightarrow{\mathrm{q}}\left(t_{n}\right)+\frac{\overrightarrow{\mathrm{p}}\left(t_{n}\right)}{m} t_{n}\right) .
\end{aligned}
$$


Recalling that in the discrete notation

$$
\int_{-\infty}^{\infty} d t \overrightarrow{\mathrm{q}} \cdot \frac{d \overrightarrow{\mathrm{p}}}{d t}-\sum_{j=1}^{n} \overrightarrow{\mathrm{q}}_{j} \cdot\left(\overrightarrow{\mathrm{p}}_{j}-\overrightarrow{\mathrm{p}}_{j-1}\right)
$$

we see that the integrals over the $\overrightarrow{\mathrm{q}}(t)$ 's for $t$ between $t_{j}$ and $t_{j-1}$ yield $\delta$ functions which equate all of the $\overrightarrow{\mathrm{p}}(t)$ 's for $t$ between $t_{j}$ and $t_{j-1}$. Remembering that in the discrete notation

$$
V\left(\overrightarrow{\mathrm{q}}\left(t_{j}\right)+\frac{\overrightarrow{\mathrm{p}}\left(t_{j}\right)}{m} t_{j}\right) \rightarrow V\left(\overrightarrow{\mathrm{q}}_{j}+\frac{\overrightarrow{\mathrm{p}}_{j}+\overrightarrow{\mathrm{p}}_{j-1}}{2 m} t_{j-1}\right)
$$

we utilize these $\delta$ functions to do some of the $\vec{p}$ integrations to obtain

$$
\begin{aligned}
S\left(\overrightarrow{\mathrm{p}}^{\prime}, \overrightarrow{\mathrm{p}}\right)=\delta^{3}\left(\overrightarrow{\mathrm{p}}-\overrightarrow{\mathrm{p}}^{\prime}\right)+\sum_{n=1}^{\infty}(-i)^{n} \int_{-\infty}^{\infty} d t_{n} \int_{-\infty}^{t_{n}} d t_{n-1} \cdots \int_{-\infty}^{t_{2}} d t_{1} \int \frac{d^{3} q_{1}}{(2 \pi)^{3}} d^{3} p_{1} \frac{d^{3} q_{2}}{(2 \pi)^{3}} \cdots d^{3} p_{n-1} \frac{d^{3} q_{n}}{(2 \pi)^{3}} & \times \exp \left[-i \sum_{j=1}^{n} \overrightarrow{\mathrm{q}}_{j} \cdot\left(\overrightarrow{\mathrm{p}}_{j}-\overrightarrow{\mathrm{p}}_{j-1}\right)\right] V\left(\overrightarrow{\mathrm{q}}_{1}+\frac{\overrightarrow{\mathrm{p}}_{1}+\overrightarrow{\mathrm{p}}_{0}}{2 m} t_{1}\right) \cdots \\
& \times V\left(\overrightarrow{\mathrm{q}}_{n}+\frac{\overrightarrow{\mathrm{p}}_{n}+\overrightarrow{\mathrm{p}}_{n-1}}{2 m} t_{n-1}\right),
\end{aligned}
$$

where $\overrightarrow{\mathrm{p}}_{0}=\overrightarrow{\mathrm{p}}$ and $\overrightarrow{\mathrm{p}}_{n}=\overrightarrow{\mathrm{p}}^{\prime}$ in the $n$th term. By changing the variables of integration from $\overrightarrow{\mathrm{q}}_{j}$ to $\overrightarrow{\mathrm{q}}_{j}+\left[\left(\overrightarrow{\mathrm{p}}_{j}+\overrightarrow{\mathrm{p}}_{j-1}\right) /\right.$ $2 m\rfloor t_{j}$, we obtain

$$
\begin{aligned}
S\left(\overrightarrow{\mathrm{p}}^{\prime}, \overrightarrow{\mathrm{p}}\right)=\delta^{3}\left(\overrightarrow{\mathrm{p}}^{\prime}-\overrightarrow{\mathrm{p}}\right)+\sum_{n=1}^{\infty}(-i)^{n} \int_{-\infty}^{\infty} d t_{n} \int_{-\infty}^{t_{n}} d t_{n-1} \cdots & \int_{-\infty}^{t_{2}} d t_{1} \\
& \times \int \frac{d^{3} q_{1}}{(2 \pi)^{3}} d^{3} p_{1} \frac{d^{3} q_{2}}{(2 \pi)^{3}} \cdots d^{3} p_{n-1} \frac{d^{3} q_{n}}{(2 \pi)^{3}} \exp \left(i \sum_{j=1}^{n} \frac{p_{j}{ }^{2}-p_{j-1}{ }^{2}}{2 m} t_{j}\right) \\
& \times \exp \left[-i \sum_{j=1}^{n} \overrightarrow{\mathrm{q}}_{j} \cdot\left(\overrightarrow{\mathrm{p}}_{j}-\overrightarrow{\mathrm{p}}_{j-1}\right)\right] V\left(\overrightarrow{\mathrm{q}}_{1}\right) \cdots V\left(\overrightarrow{\mathrm{q}}_{n}\right) .
\end{aligned}
$$

The $t$ integrations can now be done in the usual manner. Defining the $T$ matrix by

$$
S\left(\overrightarrow{\mathrm{p}}^{\prime}, \overrightarrow{\mathrm{p}}\right)=\delta^{3}\left(\overrightarrow{\mathrm{p}}^{\prime}-\overrightarrow{\mathrm{p}}\right)-2 \pi i \delta\left(\frac{p^{\prime 2}}{2 m}-\frac{p^{2}}{2 m}\right) T\left(\overrightarrow{\mathrm{p}}^{\prime}, \overrightarrow{\mathrm{p}}\right),
$$

we express our final result as

$$
T\left(\overrightarrow{\mathrm{p}}^{\prime}, \overrightarrow{\mathrm{p}}\right)=\lim _{\epsilon \rightarrow 0} \sum_{n=1}^{\infty} \int \prod_{i=1}^{n-1} d^{3} p_{i}\left(\frac{p^{2}}{2 m}-\frac{p_{i}^{2}}{2 m}+i \epsilon\right)^{-1} \prod_{i=1}^{n} \frac{d^{3} q_{i}}{(2 \pi)^{3}} V\left(\overrightarrow{\mathrm{q}}_{i}\right) \exp \left[-i \sum_{j=1}^{n} \overrightarrow{\mathrm{q}}_{j} \cdot\left(\overrightarrow{\mathrm{p}}_{j}-\overrightarrow{\mathrm{p}}_{j-1}\right)\right],
$$

where, in the $n$th term, $\overrightarrow{\mathrm{p}}_{0}=\overrightarrow{\mathrm{p}}$ and $\overrightarrow{\mathrm{p}}_{n}=\overrightarrow{\mathrm{p}}^{\prime}$. Equation (IV.6) is precisely the Born expansion for the $T$ matrix. We note that the appearances of the factor of $\delta\left(p^{\prime 2} / 2 m-p^{2} / 2 m\right)$ in Eq. (IV.5) and of the energy denominators in Eq. (IV.6) are consequences of the correspondence

$$
V\left(\ddot{\mathrm{q}}(t)+\frac{\overrightarrow{\mathrm{p}}(t)}{m} t\right)-V\left(\overrightarrow{\mathrm{q}}_{j}+\frac{\overrightarrow{\mathrm{p}}_{j}+\overrightarrow{\mathrm{p}}_{j-1}}{2 m} t_{j-i}\right)
$$

when going from the continuous to the discrete notation.

\section{PATH-INTEGRAL CALCULATION OF THE T MATRIX}

As a consequence of the energy-conserving $\delta$ function which appears in Eq. (IV.5), for given $\vec{p}$ and $\vec{p}^{\prime}, S\left(\vec{p}^{\prime}, \vec{p}\right)$ is either equal to zero or it is infinite. This fact implies that Eq. (II.8) is not directly applicable to practical calculations. In order to perform calculations, one must first extract the $\delta$ function from the $S$ matrix and then calculate the $T$ matrix; this was in fact done in the calculation of the previous section.

The manner in which the $\delta$ function arises in Eq. (II.8) can be understood in the following heuris tic way. To calculate $S\left(\vec{p}^{\prime}, \vec{p}\right)$, we integrate over all phase-space paths for which $\overrightarrow{\mathrm{p}}(t=-\infty)=\overrightarrow{\mathrm{p}}$ and $\overrightarrow{\mathrm{p}}(l=+\infty)=\overrightarrow{\mathrm{p}}^{\prime}$; the "integrand" is given by $e^{i \bar{A}}$, where $\bar{A}$ is defined by Ea. (III.5). The time translation properties of the classical interaction picture variables (see Sec. III) imply that any path can be specified by the path with $\overrightarrow{\mathrm{p}}(t=-\infty) \cdot \overrightarrow{\mathrm{q}}(t=-\infty)$ $=0$ to which it is related by a time translation and 
by the parameter $T$ of that time translation. The path in tegral can be written therefore as a double integral; one a path integral over all paths for which $\overrightarrow{\mathrm{q}}(t=-\infty) \cdot \overrightarrow{\mathrm{p}}(t=-\infty)=0$, the other an integral over $T$. We note that

$$
\tilde{A} \rightarrow \tilde{A}-\left(\frac{p^{\prime 2}}{2 m}-\frac{p^{2}}{2 m}\right) T
$$

when a time translation by an amount $T$ is performed; therefore, we expect that the integral over $T$ contains the energy conservation $\delta$ function. Therefore, in order to remove the $\delta$ function from the $S$ matrix, we integrate not over all phase-space paths, but rather only over those paths for which $\overrightarrow{\mathrm{q}}(t=-\infty) \cdot \overrightarrow{\mathrm{p}}(t=-\infty)=0$.

These considerations are reminiscent of those of Faddeev and Popov ${ }^{4}$ for the path-integral formulation of gauge-invariant field theories. In order to implement our idea, we shall use a technique which is similar to theirs.

We begin by writing "one" in the following way:

$$
1=\frac{1}{2 m}\left|\overrightarrow{\mathrm{p}}+\overrightarrow{\mathrm{p}}_{1}\right| \int_{-\infty}^{\infty} d T \delta\left(\left(\overrightarrow{\mathrm{q}}_{1}+T \frac{\overrightarrow{\mathrm{p}}+\overrightarrow{\mathrm{p}}_{1}}{2 m}\right) \cdot \frac{\overrightarrow{\mathrm{p}}+\overrightarrow{\mathrm{p}}_{1}}{\left|\overrightarrow{\mathrm{p}}+\overrightarrow{\mathrm{p}}_{1}\right|}\right) .
$$

We may insert the right-hand side of Eq. (V.1) into the integrand of Eq. (II.8) without changing anything. By interchanging the order of integration and changing variables from $\overrightarrow{\mathrm{q}}_{i}$ to $\overrightarrow{\mathrm{q}}_{i}+T\left(\overrightarrow{\mathrm{p}}_{i}+\overrightarrow{\mathrm{p}}_{i-1}\right) / 2 m$, we obtain

$$
\begin{aligned}
S\left(\overrightarrow{\mathrm{p}}^{\prime}, \overrightarrow{\mathrm{p}}\right)=\int_{-\infty}^{\infty} d T \exp \left[i\left(\frac{p^{\prime 2}}{2 m}-\frac{p^{2}}{2 m}\right) T\right] & \lim _{\tau \rightarrow \infty} \lim _{N \rightarrow \infty} \int \prod_{i=1}^{N-1} d^{3} p_{i} \prod_{i=1}^{N} \frac{d^{3} q_{i}}{(2 \pi)^{3}} \frac{\left|\overrightarrow{\mathrm{p}}+\overrightarrow{\mathrm{p}}_{1}\right|}{2 m} \delta\left(\overrightarrow{\mathrm{q}}_{1} \cdot \frac{\overrightarrow{\mathrm{p}}+\overrightarrow{\mathrm{p}}_{1}}{\left|\overrightarrow{\mathrm{p}}+\overrightarrow{\mathrm{p}}_{1}\right|}\right) \\
& \times \exp \left\{-i \sum_{j=1}^{N}\left[\overrightarrow{\mathrm{q}}_{j} \cdot\left(\overrightarrow{\mathrm{p}}_{j}-\overrightarrow{\mathrm{p}}_{j-1}\right)+\frac{2 \tau}{N} V\left(\overrightarrow{\mathrm{q}}_{j}+\frac{\left.\left.\left.\overrightarrow{\mathrm{p}}_{j}+\overrightarrow{\mathrm{p}}_{j-1}\left(t_{j-1}-T\right)\right)\right]\right\}}{2 m} .\right.\right.\right.
\end{aligned}
$$

When the $N$ and $\tau$ limits are taken, the term proportional to $T$ which appears in the potential may be set equal to zero with impunity. The $T$ integration can be done trivially; the result is given by

$$
\begin{aligned}
& S\left(\overrightarrow{\mathrm{p}}^{\prime}, \overrightarrow{\mathrm{p}}\right)=2 \pi \delta\left(\frac{p^{\prime 2}}{2 m}-\frac{p^{2}}{2 m}\right) \lim _{\tau \rightarrow \infty} \lim _{N \rightarrow \infty} \int \prod_{i=1}^{N-1} d^{3} p_{i} \prod_{i=1}^{N} \frac{d^{3} q_{i}}{(2 \pi)^{3}} \frac{\left|\overrightarrow{\mathrm{p}}+\overrightarrow{\mathrm{p}}_{1}\right|}{2 m} \delta\left(\overrightarrow{\mathrm{q}}_{1} \cdot \frac{\overrightarrow{\mathrm{p}}+\overrightarrow{\mathrm{p}}_{1}}{\left|\overrightarrow{\mathrm{p}}+\overrightarrow{\mathrm{p}}_{1}\right|}\right) \\
& \times \exp \left\{-\imath \sum_{j=1}^{N}\left[\overrightarrow{\mathrm{q}}_{j} \cdot\left(\overrightarrow{\mathrm{p}}_{j}-\overrightarrow{\mathrm{p}}_{j-1}\right)+\frac{2 \tau}{N} V\left(\overrightarrow{\mathrm{q}}_{j}+\frac{\overrightarrow{\mathrm{p}}_{j}+\overrightarrow{\mathrm{p}}_{j-1}}{2 m} t_{j-1}\right)\right]\right\} .
\end{aligned}
$$

In the case that $V=0$, the right-hand side of Eq. (V.3) must be equal to $\delta^{3}\left(\vec{p}^{\prime}-\vec{p}\right)$. We may therefore combine Eqs. (IV.5) and (V.3) to obtain the following path-integral expression for the $T$ matrix:

$$
\begin{aligned}
& T\left(\overrightarrow{\mathrm{p}}^{\prime}, \overrightarrow{\mathrm{p}}\right)=i \int\left[d^{3} p(t)\right] \int\left[\frac{d^{3} q(t)}{(2 \pi)^{3}}\right] \frac{|\overrightarrow{\mathrm{p}}+\overrightarrow{\mathrm{p}}(-\infty)|}{2 m} \delta\left(\overrightarrow{\mathrm{q}}(-\infty) \cdot \frac{\overrightarrow{\mathrm{p}}+\overrightarrow{\mathrm{p}}(-\infty)}{|\overrightarrow{\mathrm{p}}+\overrightarrow{\mathrm{p}}(-\infty)|}\right) \\
& \times \exp \left(-i \int_{-\infty}^{\infty} d t \overrightarrow{\mathrm{q}} \cdot \frac{d \overrightarrow{\mathrm{p}}}{d t}\right)\left\{\exp \left[-i \int_{-\infty}^{\infty} d t V\left(\overrightarrow{\mathrm{q}}+\frac{\overrightarrow{\mathrm{p}}}{m} t\right)\right]-1\right\} \\
& \equiv i \lim _{\tau \rightarrow \infty} \lim _{N \rightarrow \infty} \int \prod_{i=1}^{N-1} d^{3} p_{i} \prod_{i=1}^{N} \frac{d^{3} q_{i}}{(2 \pi)^{3}} \frac{\left|\overrightarrow{\mathrm{p}}+\overrightarrow{\mathrm{p}}_{1}\right|}{2 m} \delta\left(\overrightarrow{\mathrm{q}}_{1} \cdot \frac{\overrightarrow{\mathrm{p}}+\overrightarrow{\mathrm{p}}_{1}}{\left|\overrightarrow{\mathrm{p}}+\overrightarrow{\mathrm{p}}_{1}\right|}\right) \\
& \times \exp \left[-i \sum_{j=1}^{N} \overrightarrow{\mathrm{q}}_{j} \cdot\left(\overrightarrow{\mathrm{p}}_{j}-\overrightarrow{\mathrm{p}}_{j-1}\right)\right]\left\{\exp \left[-i \sum_{j=1}^{N} \frac{2 \tau}{N} V\left(\overrightarrow{\mathrm{q}}_{j}+\frac{\overrightarrow{\mathrm{p}}_{j}+\overrightarrow{\mathrm{p}}_{j-1}}{2 m} t_{j-1}\right)\right]-1\right\} .
\end{aligned}
$$

\section{EIKONAL APPROXIMATION}

The form of Eq. (V.4) suggests that the eikonal approximation can be obtained very easily from our for malism. In this section, we indicate how this comes about.

We first write the $T$ matrix as a sum of two terms:

$$
T\left(\overrightarrow{\mathrm{p}}^{\prime}, \overrightarrow{\mathrm{p}}\right)=T_{1}\left(\overrightarrow{\mathrm{p}}^{\prime}, \overrightarrow{\mathrm{p}}\right)+T_{2}\left(\overrightarrow{\mathrm{p}}^{\prime}, \overrightarrow{\mathrm{p}}\right),
$$


where

$$
T_{1}\left(\overrightarrow{\mathrm{p}}^{\prime}, \overrightarrow{\mathrm{p}}\right)=i \int\left[d^{3} p(t)\right]\left[\frac{d^{3} q(t)}{(2 \pi)^{3}}\right] \frac{|\overrightarrow{\mathrm{p}}+\overrightarrow{\mathrm{p}}(-\infty)|}{2 m} \delta\left(\overrightarrow{\mathrm{q}}(-\infty) \cdot \frac{\overrightarrow{\mathrm{p}}+\overrightarrow{\mathrm{p}}(-\infty)}{|\overrightarrow{\mathrm{p}}+\overrightarrow{\mathrm{p}}(-\infty)|}\right) \exp \left\{-i \int_{-\infty}^{\infty} d t\left[\overrightarrow{\mathrm{q}} \cdot \frac{d \overrightarrow{\mathrm{p}}}{d t}+V\left(\overrightarrow{\mathrm{q}}+\frac{\overrightarrow{\mathrm{p}}}{m} t\right)\right]\right\},
$$

and

$$
T_{2}\left(\overrightarrow{\mathrm{p}}^{\prime}, \overrightarrow{\mathrm{p}}\right)=-i \int\left[d^{3} p(t)\right]\left[\frac{d^{3} q(t)}{(2 \pi)^{3}}\right] \frac{|\overrightarrow{\mathrm{p}}+\overrightarrow{\mathrm{p}}(-\infty)|}{2 m} \delta\left(\overrightarrow{\mathrm{q}}(-\infty) \cdot \frac{\overrightarrow{\mathrm{p}}+\overrightarrow{\mathrm{p}}(-\infty)}{|\overrightarrow{\mathrm{p}}+\overrightarrow{\mathrm{p}}(-\infty)|}\right) \exp \left(-i \int_{-\infty}^{\infty} d t \overrightarrow{\mathrm{q}} \cdot \frac{d \overrightarrow{\mathrm{p}}}{d t}\right) .
$$

In the above equations for $T_{1}$ and $T_{2}$ we evaluate the $\vec{q}(-\infty)$ integral (i.e., the $\vec{q}_{1}$ integral in the discrete notation) last. The remaining integrals in Eq. (VI.3) can be evaluated easily; the result is given by

$$
T_{2}\left(\overrightarrow{\mathrm{p}}^{\prime}, \overrightarrow{\mathrm{p}}\right)=-\frac{i\left|\overrightarrow{\mathrm{p}}+\overrightarrow{\mathrm{p}}^{\prime}\right|}{2 m} \int \frac{d^{3} q_{1}}{(2 \pi)^{3}} \delta\left(\overrightarrow{\mathrm{q}}_{1} \cdot \frac{\overrightarrow{\mathrm{p}}+\overrightarrow{\mathrm{p}}^{\prime}}{\left|\overrightarrow{\mathrm{p}}+\overrightarrow{\mathrm{p}}^{\prime}\right|}\right) \exp \left[-i \overrightarrow{\mathrm{q}}_{1} \cdot\left(\overrightarrow{\mathrm{p}}^{\prime}-\overrightarrow{\mathrm{p}}\right)\right]
$$

Stationary-phase arguments show that for fixed $\vec{q}_{1}$ the major contribution to the remaining path integral in Eq. (VI.2) comes from a region centered around the classical path for which $\lim _{t \rightarrow-\alpha} \overrightarrow{\mathrm{q}}(t)=\overrightarrow{\mathrm{q}}$, and $\lim _{t \rightarrow+\infty} \overrightarrow{\mathrm{p}}(t)=\overrightarrow{\mathrm{p}}^{\prime}$. For large energy and small momentum transfer, we expect that the potential term varies slowly over this region.

We therefore approximate $V(\overrightarrow{\mathrm{q}}+\overrightarrow{\mathrm{p}} t / m)$ by $V\left(\overrightarrow{\mathrm{q}}_{\mathrm{cl}}+\overrightarrow{\mathrm{p}}_{\mathrm{cl}} t / m\right)$ in Eq. (VI.2). By $\overrightarrow{\mathrm{q}}_{\mathrm{cl}}$ and $\overrightarrow{\mathrm{p}}_{\mathrm{cl}}$ we mean the solutions of Eq. (III.4) which satisfy the boundary conditions $\lim _{t \rightarrow-\infty} \vec{q}(t)=\vec{q}_{1}$ and $\lim _{t \rightarrow+\infty} \vec{p}(t)=\vec{p}^{\prime}$. The remaining integrals in Eq. (VI.2) can be evaluated instantaneously. Combining the result with Eq. (VI.4) we obtain

$$
T\left(\overrightarrow{\mathrm{p}}^{\prime}, \overrightarrow{\mathrm{p}}\right) \approx i \frac{\left|\overrightarrow{\mathrm{p}}+\overrightarrow{\mathrm{p}}^{\prime}\right|}{2 m} \int \frac{d^{3} q_{1}}{(2 \pi)^{3}} \delta\left(\overrightarrow{\mathrm{q}}_{1} \cdot \frac{\overrightarrow{\mathrm{p}}+\overrightarrow{\mathrm{p}}^{\prime}}{\left|\overrightarrow{\mathrm{p}}+\overrightarrow{\mathrm{p}}^{\prime}\right|}\right) \exp \left[-i \overrightarrow{\mathrm{q}}_{1} \cdot\left(\overrightarrow{\mathrm{p}}^{\prime}-\overrightarrow{\mathrm{p}}\right)\right]\left\{\exp \left[-i \int_{-\infty}^{\infty} d t V\left(\overrightarrow{\mathrm{q}}_{\mathrm{cl}}+\frac{\overrightarrow{\mathrm{p}}_{\mathrm{cl}}}{2 m} t\right)\right]-1\right\} .
$$

Investigation of Eq. (III.4) shows that for large energy $\overrightarrow{\mathrm{q}}(t)-\overrightarrow{\mathrm{q}}_{1}=O\left(1 / p^{\prime 2}\right)$ and $\overrightarrow{\mathrm{p}}_{\mathrm{cl}}(t)-\overrightarrow{\mathrm{p}}^{\prime}=O\left(1 /\left|p^{\prime}\right|\right)$. Further more, energy conservation implies that $\left|\overrightarrow{\mathrm{p}}_{\mathrm{cl}}(t)\right|-|\overrightarrow{\mathrm{p}}|=O\left(1 / p^{\prime 2}\right)$. We may therefore make the further approximation: $\overrightarrow{\mathrm{q}}_{\mathrm{cl}}(t)=\overrightarrow{\mathrm{q}}_{1}$ and $\overrightarrow{\mathrm{p}}_{\mathrm{cl}}(t)=\left|\overrightarrow{\mathrm{p}}^{\prime}\right| \hat{n}$, where $\hat{n}$ is a suitably chosen unit vector, in Eq. (VI.5). We may evaluate the integration over the component of $\vec{q}_{1}$ parallel to $\vec{p}+\vec{p}^{\prime}$ to obtain

$$
T\left(\overrightarrow{\mathrm{p}}^{\prime}, \overrightarrow{\mathrm{p}}\right) \approx i \frac{\left|\overrightarrow{\mathrm{p}}+\overrightarrow{\mathrm{p}}^{\prime}\right|}{2 m} \int \frac{d^{2} b}{(2 \pi)^{3}} \exp \left[-i \overrightarrow{\mathrm{b}} \cdot\left(\overrightarrow{\mathrm{p}}^{\prime}-\overrightarrow{\mathrm{p}}\right)\right]\left\{\exp \left[-i \int_{-\infty}^{\infty} d t V\left(\overrightarrow{\mathrm{b}}+\frac{|\overrightarrow{\mathrm{p}}| \hat{n}}{m} t\right)\right]-1\right\} .
$$

In Eq. (VI.6) $\vec{b}$ ranges over the plane perpendicular to $\vec{p}+\vec{p}^{\prime}$ with $\vec{q} \cdot\left(\vec{p}+\vec{p}^{\prime}\right)=0$. Equation (VI.6) is essentially the eikonal approximation ${ }^{5}$; various forms for the eikonal approximation are obtained by suitable choices for $n$.

Higher-order approximations may be obtained by expanding $V(\vec{q}+\vec{p} t / m)$ in a Taylor series around the point $V\left(\overrightarrow{\mathrm{q}}_{\mathrm{cl}}+\overrightarrow{\mathrm{p}}_{\mathrm{cl}} t / m\right)$ and retaining a finite number of terms. The first correction in Eq. (VI.5) can be obtained very simply. In the discrete form of Eq. (VI.2) we approximate $V\left(\vec{q}_{j}+\left(\vec{p}_{j}+\vec{p}_{j-1}\right) t_{j-1} / m\right)$ by

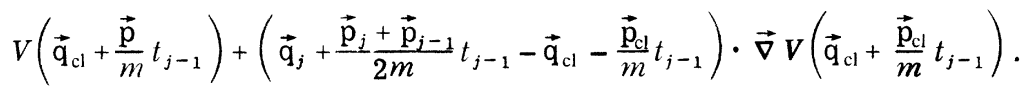

Equation (VI.2) is thereby approximated by

$$
\begin{aligned}
T_{1} \approx i \lim _{\tau \rightarrow \infty} \lim _{N \rightarrow \infty} \int \prod_{i=1}^{N-1} d^{3} p_{i} \prod_{i=1}^{N} & \frac{d^{3} q_{i}}{(2 \pi)^{3}} \frac{\left|\overrightarrow{\mathrm{p}}+\overrightarrow{\mathrm{p}}_{1}\right|}{2 m} \delta\left(\overrightarrow{\mathrm{q}}_{1} \cdot \frac{\overrightarrow{\mathrm{p}}+\overrightarrow{\mathrm{p}}_{1}}{\left|\overrightarrow{\mathrm{p}}+\overrightarrow{\mathrm{p}}_{1}\right|}\right) \\
& \times \exp \left\{-i \sum_{j=1}^{N} \overrightarrow{\mathrm{q}}_{j} \cdot\left[\overrightarrow{\mathrm{p}}_{j}-\overrightarrow{\mathrm{p}}_{j-1}+\frac{2 \tau}{N} \vec{\nabla} V\left(\overrightarrow{\mathrm{q}}_{\mathrm{cl}}+\frac{\overrightarrow{\mathrm{p}}_{\mathrm{cl}}}{m} t_{j-1}\right)\right]\right\} \\
& \times \exp \left\{i \sum _ { j = 1 } ^ { N } \frac { 2 \tau } { N } t _ { j - 1 } \left[\frac{\left.\left.\overrightarrow{\mathrm{p}}_{j}+\overrightarrow{\mathrm{p}}_{j-1}-2 \overrightarrow{\mathrm{p}}_{\mathrm{cl}} \cdot \vec{\nabla} V\left(\overrightarrow{\mathrm{q}}_{\mathrm{cl}}+\frac{\overrightarrow{\mathrm{p}}_{\mathrm{cl}}}{m} t_{j-1}\right)\right]\right\}}{2 m}\right.\right. \\
& \times \exp \left\{-i \int_{-\infty}^{\infty} d t\left[V\left(\overrightarrow{\mathrm{q}}_{\mathrm{cl}}+\frac{\overrightarrow{\mathrm{p}}_{\mathrm{cl}}}{m} t\right)-\overrightarrow{\mathrm{q}}_{\mathrm{cl}} \cdot \vec{\nabla} V\left(\overrightarrow{\mathrm{q}}_{\mathrm{cl}}+\frac{\overrightarrow{\mathrm{p}}_{\mathrm{cl}}}{m} t\right)\right]\right\}
\end{aligned}
$$


The $\overrightarrow{\mathrm{q}}_{j}$ integral yields a factor of

$$
\delta^{3}\left(\overrightarrow{\mathrm{p}}_{j}-\overrightarrow{\mathrm{p}}_{j-1}+\frac{2 \tau}{N} \vec{\nabla} V\left(\overrightarrow{\mathrm{q}}_{\mathrm{cl}}+\frac{\overrightarrow{\mathrm{p}}_{\mathrm{c}}}{m} t_{j-1}\right)\right)
$$

for $j=2, \ldots, N$; in the limit $N \rightarrow \infty$, this factor becomes $\delta^{3}\left(d p+d t V\left(\overrightarrow{\mathrm{q}}+\left(\overrightarrow{\mathrm{p}}_{\mathrm{cl}} / m\right) t\right)\right)$. Therefore, when the $\overrightarrow{\mathrm{p}}_{j}$ integral is carried out, $\overrightarrow{\mathrm{p}}_{j}$ is to be set equal to $\overrightarrow{\mathrm{p}}_{\mathrm{cl}}\left(t_{j}\right)$. We can therefore reduce Eq. (VI.7) to the form

$$
T_{1}\left(\overrightarrow{\mathrm{p}}^{\prime}, \overrightarrow{\mathrm{p}}\right) \approx i \int \frac{d^{3} q_{1}}{(2 \pi)^{3}} \frac{\left|\overrightarrow{\mathrm{p}}+\overrightarrow{\mathrm{p}}_{\mathrm{cl}}(-\infty)\right|}{2 m} \delta\left(\overrightarrow{\mathrm{q}}_{1} \cdot \frac{\overrightarrow{\mathrm{p}}+\overrightarrow{\mathrm{p}}_{\mathrm{cl}}(-\infty)}{\left|\overrightarrow{\mathrm{p}}+\overrightarrow{\mathrm{p}}_{\mathrm{cl}}(-\infty)\right|}\right) \exp \left[i \tilde{A}_{\mathrm{cl}}\left(\overrightarrow{\mathrm{q}}_{1}, \overrightarrow{\mathrm{p}}^{\prime}\right)\right] \exp \left\{-i \overrightarrow{\mathrm{q}}_{1} \cdot\left[\overrightarrow{\mathrm{p}}_{\mathrm{cl}}(-\infty)-\overrightarrow{\mathrm{p}}\right]\right\} .
$$

In deriving Eq. (VI.8) we have used the classical equations of motion Eq. (III.4) and the definition of $\tilde{A}$, Eq. (III.5). In Eq. (VI.8) $\tilde{A}\left(\overrightarrow{\mathrm{q}}_{1}, \overrightarrow{\mathrm{p}}^{\prime}\right)$ is the value of the "action" for the classical path specified by the boundary conditions $\overrightarrow{\mathrm{q}}(-\infty)=\overrightarrow{\mathrm{q}}_{1}$ and $\overrightarrow{\mathrm{p}}(+\infty)=\overrightarrow{\mathrm{p}}^{\prime}$.

*Permanent address: Behlen Laboratory of Physics, University of Nebraska, Lincoln, Nebraska 68508.

tWork supported in part by the University of Nebraska Research Council.

\$Work supported in part by NSF Grant No. GP-43907.

${ }^{1}$ R. P. Feynman and A. R. Hibbs, Quantum Mechanics and Path Integrals (McGraw-Hill, New York, 1965).

${ }^{2}$ See e.g., E. S. Abers and B. W. Lee, Phys. Rep. 9C,
1 (1973).

${ }^{3}$ W. B. Campbell, P. Finkler, C. E. Jones, and M. N. Misheloff, University of Nebraska report (unpublished).

${ }^{4}$ V. M. Popov and L. D. Faddeev, Kiev ITP report (unpublished).

${ }^{5}$ See, M. L. Goldberger and K. M. Watson, Collision Theory (Wiley, New York, 1964), pp. 330-339. 